\title{
Clinical Study \\ Effects of Neuromuscular Electrical Stimulation Combined with Exercises versus an Exercise Program on the Pain and the Function in Patients with Knee Osteoarthritis: A Randomized Controlled Trial
}

\author{
Aline Mizusaki Imoto, ${ }^{1}$ Stella Peccin, ${ }^{1,2}$ \\ Kelson Nonato Gomes da Silva, ${ }^{1}$ Lucas Emmanuel Pedro de Paiva Teixeira, ${ }^{1}$ \\ Marcelo Ismael Abrahão, ${ }^{1}$ and Virgínia Fernandes Moça Trevisani ${ }^{1,3}$ \\ ${ }^{1}$ Internal Medicine Department, Federal University of São Paulo, 04023-900 São Paulo, SP, Brazil \\ ${ }^{2}$ Human Movement Science Department, Federal University of São Paulo, 11060-001 Santos, SP, Brazil \\ ${ }^{3}$ Medicine Department, Santo Amaro University, 04829-300 São Paulo, SP, Brazil \\ Correspondence should be addressed to Aline Mizusaki Imoto; aline.mizusaki@globo.com
}

Received 23 April 2013; Revised 10 August 2013; Accepted 12 August 2013

Academic Editor: Jón Karlsson

Copyright (C) 2013 Aline Mizusaki Imoto et al. This is an open access article distributed under the Creative Commons Attribution License, which permits unrestricted use, distribution, and reproduction in any medium, provided the original work is properly cited.

\begin{abstract}
Objectives. To investigate the effect of 8 weeks of NMES + Ex (neuromuscular electrical stimulation combined with exercises) on pain and functional improvement in patients with knee osteoarthritis (OA) compared to exercise (Ex) alone. Design. Randomized controlled trial. Setting. A specialty outpatient clinic. Participants. Patients $(N=100$; women $=86$, $\operatorname{men}=14$; age range, $50-75$ years $)$ with knee OA. Interventions. Participants were randomly assigned to NMES + Ex or Ex group. Outcome Measures. Numerical Rating Scale 0 to 10 (NRS) and the Timed Up and Go (TUG) test were the primary outcomes. The secondary outcomes used were the Western Ontario and McMaster Universities Osteoarthritis Index (WOMAC). Results. Following the interventions, a statistically significant improvement in both groups was observed in all outcomes assessed. For the comparison between the groups, no statistically significant difference was found between the NMES + Ex and the Ex groups in NRS $(P=0.52)$, TUG test $(P=0.12)$, and aspects of WOMAC: pain $(P=0.26)$, function $(P=0.23)$, and stiffness $(P=0.63)$. Conclusion. The addition of NMES to exercise did not improve the outcomes assessed in knee OA patients. This study was registered at the Australian Clinical Trials Registry (ACTRN012607000357459).
\end{abstract}

\section{Introduction}

Osteoarthritis (OA) is the most common form of arthritis. It affects one-third of adults and tends to increase with age [1]. Knee OA is associated with symptoms of pain, swelling, instability, and reduced range of motion (ROM). These symptoms lead to functional impairment, increasing the risk of morbidity and mortality $[2,3]$.

The synovium is infiltrated with inflammatory cells and secretes excess synovial fluid, leading to capsular swelling [4]. Through a spinal reflex, the capsular swelling inhibits muscle activation, which, combined with disuse, may cause muscle weakness and atrophy [1]. Because the quadriceps muscle acts as shock absorber for the knee joint, weakness in the thigh muscle reduces joint protection, resulting in overload [4]. Exercises strengthen the muscles, reduce pain, improve physical function, and are therefore considered a major intervention in the conservative treatment of patients with knee $\mathrm{OA}$ [5]. In addition to muscle strengthening exercises, stretching exercises are commonly used to increase ROM and are often prescribed in rehabilitation protocols as part of routine warm-up to prepare the muscles and joints for other types of exercise, such as aerobic and strengthening programs $[6,7]$. Stretching of the hamstring muscles may improve knee extension ROM in OA patients. 
Neuromuscular electrical stimulation (NMES) is defined as the application of electrical stimulation using surface electrodes placed over skeletal muscles to produce visible muscle contraction through the activation of intramuscular nerve branches [8]. This technique can also be used as a form of physical therapy in the treatment of patients with knee OA. The goals of rehabilitation protocols that include NMES are to provide additional stimulus to increase muscle strength in patients with knee OA [9]. The methods and findings of previous studies on the effectiveness of NMES in knee OA differ in the modulation of NMES parameters, choice of the outcomes used to evaluate the patients, and characteristics of the control groups. This leads to a lack of consensus regarding the effectiveness attained from including NMES in conventional rehabilitation protocols. Major flaws with regard to methodological quality were found in clinical trials testing the use of NMES in the conservative treatment of patients with knee OA. Only one of these studies reported using allocation concealment, blinded assessment, and intention-to-treat analysis [10].

Thus, the objective of this study was to conduct a randomized clinical trial following methodological criteria, including allocation concealment, blinding of the examiner, and application of intention-to-treat analysis to assess the role of NMES in improving pain and physical function in patients with knee OA. It is important to study interventions with the potential to improve functional status in this patient population [11].

\section{Methods}

The study was conducted at the Interlagos Specialty Outpatient Clinic, São Paulo, Brazil. Patients were referred from the Rheumatology Department according to the inclusion and exclusion criteria and randomly allocated into groups using a computer-generated randomization chart. The allocation codes were sealed in opaque envelopes by a third person not involved in the study to avoid selection bias.

Written informed consent was obtained from all participants. The study was approved by the Research Ethics Committee of the Universidade Federal de São Paulo (UNIFESP), Brazil, no. 0141/07, and registered with the Australian Clinical Trials Registry, no. ACTRN012607000357459.

Setting the significance level at $5 \%$ and the power of the sample at $80 \%$, a sample size of 40 patients per group was estimated to be necessary to detect a difference of at least 1 minute \pm 3 seconds in the Timed Up and Go (TUG) test, which was considered to be the minimum clinically significant difference for the present trial [12]. Paired Student's $t$-test and analysis of covariance (ANCOVA) were used for comparisons between groups; the covariant was obtained from a previous study [13].

One hundred patients were recruited according to inclusion and exclusion criteria. Eligibility criteria were age 50 to 75 years, OA grade 2 or greater according to the radiographic classification of OA proposed by Kellgren and Lawrence [14], and diagnosis of knee OA based on the American College of Rheumatology (ACR) criteria. Exclusion criteria were use of a pacemaker, unstable heart conditions, participation in another physical activity program, inability to exercise on a stationary bicycle ergometer, inability to walk, previous hip or knee arthroplasty, diagnosis of fibromyalgia, epilepsy, and skin tumor or lesion at the NMES application site.

Patients were divided into two groups of 50 each: (1) the NMES combined with exercises (NMES + Ex) group and (2) exercise (Ex) group. Patient medication was standardized and not modified during the study period. Paracetamol was prescribed for pain, and diacerein and chloroquine for OA control.

Interventions were delivered for both groups by the same physical therapist, twice a week, for 8 weeks, with each session lasting about 40 minutes.

All patients received a manual including guidelines on how not to overload the knee during daily activities and instructions on the use of ice packs in case of pain and inflammation and warm compresses in case of pain without inflammation as follows.

Manual for Patients with Osteoarthritis of the Knee. The purpose of this manual is to explain osteoarthritis and to teach how you can adjust yourself to your daily activities, according to the knee symptoms.

Try to seriously follow our orientations for your own benefit!

The Knee. The knee joint is composed of 3 bones-the femur (thigh bone), the patella (kneecap) and the tibia (leg bone). It has muscles, capsule, ligaments, meniscus, and the cartilage that lines the bones and protects them from the impact. The knee joint supports nearly the whole weight of our body.

What Is Osteoarthritis? It is a disease caused by the breakdown of cartilage in the joints. The layers in the cartilage become damaged and with time they lose the function of smoothing the contact between the bone surface and the joints. The pain is a result of the attrition of one bone against the other in the absence or decreased cartilage in the joints.

What Are the Signs and Symptoms? Patients with osteoarthritis may have some pain mainly when starting a movement, as in morning stiffness or after immobilization. With time, the pain might be intensified and be permanent. The presence of crepitation when moving the knees is often.

What Kind of Difficulties Might I Have in My Daily Life? Difficulties found in daily live vary according to the patients' symptoms. In general, however, the patient has pain and difficulty when supporting the body weight using the affected knee, going up and down the stairs, or when walking.

What Should I Do When It Is Painful? A doctor can prescribe the treatment for osteoarthritis. However, a simple form of improving the pain is to use warm to hot water bottle over the knee joint (be careful not to burn the skin, use a protection, and test the water temperature before using it.

What If It Is Swollen? To manage the swollen, you can combine rest, use of ice pack, and elevating the leg above the level of the heart. The ice pack should be placed over the knee joint for 20 minutes. 


\section{What Are the Other Recommendations?}

(i) If you are overweight, losing some kilos will reduce the stress over the joint.

(ii) Wear comfortable shoes with a rubber sole and no heels.

(iii) In case of pain when walking, use a cane as an aid tool.

(iv) Try to have a good night sleep.

2.1. NMES Combined with Exercise Group. Treatment for patients in the NMES + Ex included 10 minutes on a stationary bicycle, stretching of hamstring muscles (3 repetitions of 30 seconds) with the aid of an elastic band, and loaded quadriceps strengthening exercises combined with NMES. The strengthening exercise with NMES was performed in the sitting position with the knee and hip flexed to 90 degrees; patients contracted their quadriceps at each NMES stimulus.

NMES was applied using an electrical stimulator (Globus ACTIVA 600 Pro, Globus, Italia) with two $7.5 \times 13 \mathrm{~cm}$ selfadhesive electrodes (ValuTrode electrodes, Axelgaard Manufacturing Co. Ltd., Fallbrook, CA) placed over the region of the quadriceps muscle (rectus femoris and vastus medialis). NMES parameters were as follows: pulsed current, biphasic, asymmetrical, rectangular waveform, frequency $50 \mathrm{~Hz}$, pulse duration $250 \mu \mathrm{s}$, contraction time $10 \mathrm{~s}$, rest time $30 \mathrm{~s}$ every 20 minutes; current intensity was the maximum tolerated by each patient [15].

2.2. Exercise Group (Ex). Patients in the Ex group performed the same exercise program as those in the NMES group but without NMES. The exercise protocol included 10 minutes of warm-up on a stationary bicycle ergometer, stretching of hamstrings muscles with the aid of an elastic band, and knee extension exercises performed for 3 sets of 15 repetitions with rest intervals of 30-45 seconds between sets.

For both groups, the training load for the strengthening exercises was established based on $50-60 \%$ of the 10 -repetition maximum (RM) instead of $1 \mathrm{RM}$ to avoid injury by excessive muscle contraction [16].

2.3. Outcomes. Patients were evaluated before and after intervention by a physical therapist blinded to group assignment. The primary outcomes were the TUG test results [12] and pain walking on a flat surface in the last $72 \mathrm{~h}$ measured on an 11-point Numerical Rating Scale (NRS) [17]. The secondary outcomes were scores on the pain, physical function, and stiffness subscales of the culturally validated BrazilianPortuguese version of the Western Ontario and McMaster Universities Osteoarthritis Index (WOMAC) [18, 19]. In this study, WOMAC pain, physical function, and stiffness scores were analyzed separately.

2.4. Statistical Analysis. Paired Student's t-test was performed to compare pre- and postintervention values at a significance level of $0.05(P<0.05)$. Statistical analysis was performed on an intent-to-treat (ITT) basis and included all patients who were randomized to treatment. Mixed model analysis of variance with repeated measures was used with occasion
TABLE 1: Baseline characteristics of subjects.

\begin{tabular}{lcc}
\hline Characteristics & NMES + Ex & Ex \\
\hline$N$ & 50 & 50 \\
Age $^{*}$ & $60.60 \pm 6.72$ & $61.50 \pm 6.94$ \\
Female $^{\dagger}$ & 46 & 40 \\
Male $^{\dagger}$ & 4 & 10 \\
Treated leg $^{\dagger}$ & & \\
$\quad$ Right & 40 & 40 \\
$\quad$ Left & 34 & 34 \\
$\quad$ Both sides & 26 & 26 \\
BMI $^{*}$ & $30.08 \pm 3.80$ & $29.72 \pm 4.11$ \\
KL grade $^{\dagger}$ & & \\
$\quad 2$ & 95.35 & 92.68 \\
$\quad 3$ & 2.33 & 4.88 \\
$\quad 4$ & 2.33 & 2.44 \\
NRS (0-10) $^{*}$ & $7.06 \pm 1.95$ & $7.42 \pm 2.01$ \\
TUG Test (seconds) $^{*}$ & $8.27 \pm 1.76$ & $9.34 \pm 2.47$ \\
WOMAC pain score $^{*}$ & $8.72 \pm 4.20$ & $10.32 \pm 3.54$ \\
WOMAC stiffness score $^{*}$ & $3.64 \pm 2.15$ & $3.66 \pm 2.64$ \\
WOMAC function score $^{*}$ & $28.54 \pm 13.96$ & $35.15 \pm 11.88$ \\
\hline
\end{tabular}

Note. ${ }^{*}$ Data are presented as mean $\pm \mathrm{SD} .{ }^{\dagger}$ Data are presented as \%. Abbreviations: BMI: body mass index, KL: Kellgren and Lawrence; NRS: Numerical Rating Scale; TUG: Timed Up and Go; WOMAC: Western Ontario and Mcmaster Universities. NMES + Ex: NMES combined with exercise, Ex: Exercise, NRS: Numerical Rating Scale; TUG: Timed Up and Go Test, WOMAC: Western Ontario McMaster Universities Osteoarthritis Index.

measures as within-group factors and intervention as between-group factor.

Relations between the observations were analyzed using an unstructured covariance matrix. Missing-data imputation was not performed to evaluate pre- and postintervention differences between the two groups, because Chakraborty and GU [20] showed that mixed model analysis without missingdata imputation always provides equal or more power than does mixed model analysis with missing-data imputation. Effect size was calculated as the difference between the means divided by the standard deviation using Cohen's $d$ [21]. The analyses were performed using the General Linear Model (GLM), and mixed analyses were carried out using the Statistical Analysis Software (SAS) version 9.2 for Windows [22].

\section{Results}

The demographic and clinical characteristics of patients, including age, sex, side treated, and body mass index (BMI), as well as TUG test values, NRS pain scores, and WOMAC scores on the pain, physical function, and stiffness subscales, are shown in Table 1. Eighty-seven patients completed the study. Of the 13 dropouts, 6 (12\%) patients were in the NMES + Ex group and 7 (14\%) in the Ex group (Figure 1).

3.1. Primary Outcomes. No significant differences between groups were found in NRS pain scores and TUG test time on ITT analysis. A significant decrease in pain intensity (NRS 


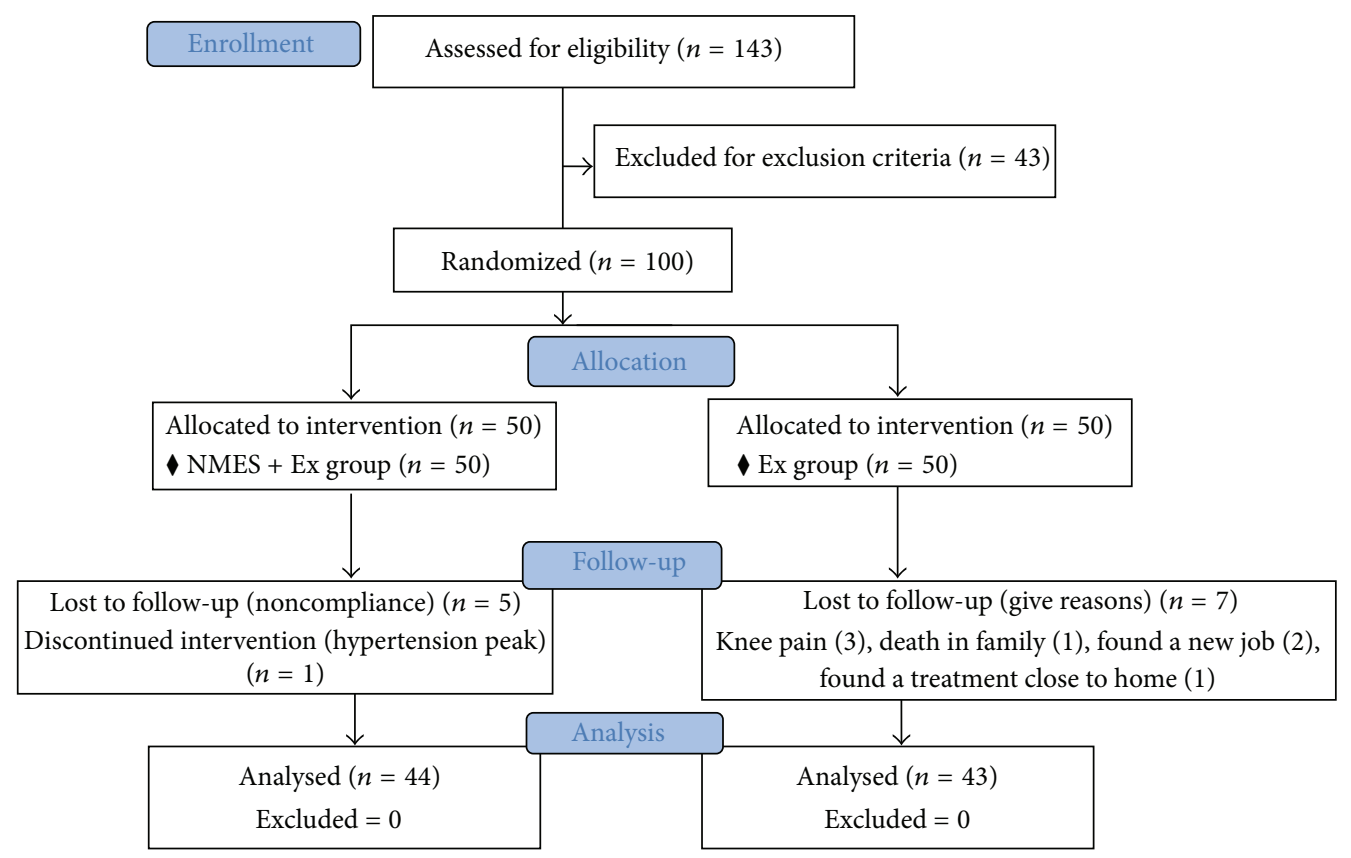

FIGURE 1: Flowchart showing the number of patients randomized and evaluated in each group.

TABLE 2: Changes within and between groups (ITT analysis).

\begin{tabular}{|c|c|c|c|c|c|c|}
\hline \multirow{2}{*}{ Outcomes } & \multicolumn{2}{|r|}{ NMES + Ex } & \multicolumn{2}{|r|}{ Ex } & \multirow{2}{*}{$\begin{array}{l}\text { Difference between means } \\
(95 \% \text { CI }) \text {, effect size }\end{array}$} & \multirow{2}{*}{$\begin{array}{l}P \text { value between } \\
\text { groups }\end{array}$} \\
\hline & At 8 weeks & Change (95\% CI) & At 8 weeks & Change (95\% CI) & & \\
\hline NRS $(0-10)^{*}$ & $4.30 \pm 3.01$ & $-2.70(-3.56 \text { to }-1.84)^{\dagger}$ & $4.27 \pm 2.45$ & $-3.17(-4.23 \text { to }-2.10)^{\dagger}$ & $\begin{array}{c}0.42(-0.87 \text { to } 1.72) \\
0.15\end{array}$ & 0.52 \\
\hline TUG $^{*}$ & $6.77 \pm 1.08$ & $-1.36(-1.84 \text { to }-0.87)^{\dagger}$ & $7.42 \pm 1.70$ & $-2.00(-2.54 \text { to }-1.46)^{\dagger}$ & $\begin{array}{c}0.56(-0.15 \text { to } 1.27) \\
0.39\end{array}$ & 0.12 \\
\hline $\begin{array}{l}\text { WOMAC } \\
\text { pain* }^{*}\end{array}$ & $5.64 \pm 4.33$ & $-2.97(-4.22 \text { to }-1.72)^{\dagger}$ & $6.29 \pm 3.96$ & $-3.87(-5.02 \text { to }-2.72)^{\dagger}$ & $\begin{array}{c}0.92(-0.71 \text { to } 2.55) \\
0.23\end{array}$ & 0.26 \\
\hline $\begin{array}{l}\text { WOMAC } \\
\text { stiffness }^{*}\end{array}$ & $2.39 \pm 2.24$ & $-1.34(-1.9 \text { to }-0.74)^{\dagger}$ & $2.10 \pm 2.26$ & $-1.51(-2.36 \text { to }-0.65)^{\ddagger}$ & $\begin{array}{c}0.25(-0.78 \text { to } 1.29) \\
0.10\end{array}$ & 0.63 \\
\hline $\begin{array}{l}\text { WOMAC } \\
\text { function* }\end{array}$ & $20.91 \pm 14.06$ & $-8.02(-11.34 \text { to }-4.69)^{\dagger}$ & $23.83 \pm 15.49$ & $-10.95(-14.84 \text { to }-7.05)^{\dagger}$ & $\begin{array}{l}3.14(-2.02 \text { to } 8.29) \\
0.26\end{array}$ & 0.23 \\
\hline
\end{tabular}

Abbreviation: ${ }^{*}$ data are presented as mean \pm SD. ITT: intention to treat; CI: confidence interval; NMES + Ex: NMES combined with exercise, Ex: exercise; NRS: Numerical Rating Scale; TUG: Timed Up and Go Test; WOMAC: Western Ontario McMaster Universities; ${ }^{\dagger} P<0.05$ denotes a statistical significant difference, ${ }^{\ddagger} P<0.0001$.

scores) and TUG test time was observed after intervention compared with baseline in the NMES + Ex group (NRS scores, $P<0.0001$; TUG test, $P<0.0001$ ) and Ex group (NRS scores, $P<0.0001$; TUG test, $P<0.0001)$. The primary outcomes are listed in Table 2.

3.2. Secondary Outcomes. No significant differences between groups were found on the pain, physical function, and stiffness subscales of the WOMAC index on ITT analysis. There was a significant improvement in all WOMAC subscales in the NMES + Ex group (pain; $P<0.0001$; physical function, $P<0.0001$; stiffness, $P<0.0001$ ) and in the Ex group (pain, $P<0.0001$; physical function, $P<0.0001$; stiffness, $P=$ $0.0009)$. The secondary outcomes are listed in Table 2.

3.3. Adverse Effect. One patient in the NMES + Ex group exhibited a blood pressure spike, which may have resulted from the use of NMES or from the exercise program itself. The following contraindications to the use of NMES were respected: avoiding the use of NMES over areas of tumor, with open wounds, or bleeding, and in patients with pacemakers [15].

\section{Discussion}

In this randomized clinical trial, the NMES combined with exercise did improve pain and physical function, but there was no evidence that it did better than exercise alone. Our results are in agreement with the findings of Rosemffet et al. [23] who conducted a pilot study comparing the treatment results of patients with knee OA $(n=26)$ treated with either NMES alone or exercises alone or exercises combined with NMES. The authors reported a significant improvement on the WOMAC pain subscale in the three groups, but 
no significant differences in WOMAC scores were found between groups [23].

The lack of difference in treatment outcome between groups in the present study might be attributed to the fact that the participants had no clinically significant muscle or functional impairment. A finding substantiating this hypothesis is that the mean TUG test values in the two groups were similar to those found in the study of Steffen et al., for elderly patients with no physical limitations categorized under the same mean age group [24]. The TUG test values reported were $8 \pm 2$ seconds for patients with 60-69 years old and $9 \pm 3$ seconds for patients with 70-79 years old. In the present study, the baseline values for TUG test were $8.27+1.76$ for NMES + Ex group and $9.34 \pm 2.47$ for Ex group.

Given that the greater the muscle impairment, the greater the NMES effect, patients with a more advanced stage of OA might obtain greater benefit from NMES [25-27]. With regard to evidence on the effectiveness of NMES in the rehabilitation of patients with knee OA, the Cochrane Collaboration published a systematic review evaluating the pre- and postoperative use of NMES in total knee arthroplasty [28]. The two studies that were evaluated were classified as being at high risk of bias, because randomization and allocation concealment were not described, and no data obtained from the study groups were presented in terms of means and standard deviations. It was not possible to determine whether or not the pre- and postoperative use of NMES was effective in the rehabilitation of knee arthroplasty patients, and therefore further studies are warranted. Regarding lines of evidence on the effectiveness of NMES in the treatment of other conditions, Kim et al. [29] conducted a review of the use of NMES after anterior cruciate ligament (ACL) reconstruction. Based on eight different studies, the authors concluded that the use of NMES combined with exercises can be effective in improving quadriceps strength up to four weeks after surgery. However, there was no sufficient evidence to affirm that NMES has any positive effect on the functional performance of patients who had undergone ACL reconstruction [29]. No literature review could be found on the effectiveness of NMES in patients with knee OA without indication for knee arthroplasty.

A limitation of this study is that the current intensity used for electrical stimulation was not recorded. However, the maximum current intensity tolerated by each patient was applied as in previous studies [9,24,30]. No participant showed intolerance to electrical stimulation.

The questionnaires used in this study have been translated into Brazilian Portuguese, cross-culturally adapted, and validated in previous studies $[19,31,32]$. The assessment of patient-reported pain intensity and physical function was performed according to the international consensus on outcomes measures for phase III clinical trials in OA, in which pain and physical function are identified as the most important outcome measures in randomized clinical trials [18]. The TUG test was chosen because it is a simple and inexpensive test designed to assess the functional mobility of patients based on activities of daily living [12].

Statistical analysis was performed on an intent-totreat basis to minimize the impact of protocol violations (which may occur after randomization) on the results and conclusions and to avoid an overestimation of the treatment effect. This randomized clinical trial conforms to the Consort Statement (Consolidated Standards of Reporting Trials) [33], whose aim is to improve the quality of reports of randomized clinical trials. Given the predominance of mild and moderate OA cases among the participants, the results of this study can be generalized especially for patients with similar severity levels of OA.

\section{Conclusions}

Our results revealed that the application of NMES combined with a conventional exercise program was as effective as the exercise program alone in reducing pain and improving physical function in patients with knee OA, and therefore no therapeutic benefit was observed with the use of NMES.

\section{Clinical Implications}

Moderate exercises, including warm-up and muscle stretching and strengthening exercises-combined or not with NMES-are recommended to reduce pain and improve physical function and quality of life in patients with knee OA.

\section{List of Abbreviations}

$\begin{array}{ll}\text { ACTRN: } & \text { Australian Clinical Trials Registry Number } \\ \text { ACR: } & \text { American College of Rheumatology } \\ \text { ANCOVA: } & \text { Analysis of covariance } \\ \text { BMI: } & \text { Body mass index } \\ \text { CONSORT: } & \text { Consolidated standards of reporting trials } \\ \text { Ex: } & \text { Exercises } \\ \text { GLM: } & \text { General linear models } \\ \text { ITT: } & \text { Intention-to-treat analysis } \\ \text { NMES + Ex: } & \text { Neuromuscular electrical stimulation } \\ & \text { combined with exercises } \\ \text { NRS: } & \text { Numerical Rating Scale } \\ \text { NMES: } & \text { Neuromuscular electrical stimulation } \\ \text { OA: } & \text { Osteoarthritis } \\ \text { RM: } & \text { Repetition maximum } \\ \text { ROM: } & \text { Range of motion } \\ \text { SAS: } & \text { Statistical analysis software } \\ \text { SD: } & \text { Standard deviation } \\ \text { TUG: } & \text { Timed Up and Go } \\ \text { UNIFESP: } & \text { Universidade Federal de Sao Paulo } \\ \text { WOMAC: } & \text { Western Ontario and McMaster Universities } \\ & \text { Osteoarthritis Index. }\end{array}$

\section{Acknowledgments}

This study was supported by Fundação de Apoio a Pesquisa do Estado de São Paulo-FAPESP. The authors express their gratitude to Dr. Milton Mizsputen, responsible for the radiographic classification; the head supervisor of the Specialty Ambulatory of Interlagos, and Brazil Cochrane Center. They certify that no party having a direct interest in the results of the research supporting this paper has or will confer a benefit on them or on any organization with which they are associated and, if applicable, they certify that all financial and 
material support for this research (e.g., NIH or NHS grants) and work are clearly identified in the title page of the paper.

\section{References}

[1] D. T. Felson, "Developments in the clinical understanding of osteoarthritis," Arthritis Research and Therapy, vol. 11, no. 1, article 203, 2009.

[2] B. T. Maurer, A. G. Stern, B. Kinossian, K. D. Cook, and H. R. Schumacher Jr., "Osteoarthritis of the knee: isokinetic quadriceps exercise versus and educational intervention," Archives of Physical Medicine and Rehabilitation, vol. 80, no. 10, pp. 12931299, 1999.

[3] T. S. Fidelix, B. G. Soares, and V. F. Trevisani, "Diacerein for osteoarthritis," Cochrane Database of Systematic Reviews, no. 1, Article ID CD005117, 2006.

[4] M. V. Hurley, "The role of muscle weakness in the pathogenesis of osteoarthritis," Rheumatic Disease Clinics of North America, vol. 25, no. 2, pp. 283-298, 1999.

[5] S. P. Messier, R. F. Loeser, G. D. Miller et al., "Exercise and dietary weight loss in overweight and obese older adults with knee osteoarthritis: the Arthritis, Diet, and Activity Promotion Trial," Arthritis and Rheumatism, vol. 50, no. 5, pp. 1501-1510, 2004.

[6] D. A. Reid and P. J. McNair, "Effects of an acute hamstring stretch in people with and without osteoarthritis of the knee," Physiotherapy, vol. 96, no. 1, pp. 14-21, 2010.

[7] J. P. K. Halbertsma, I. Mulder, L. N. H. Göeken, and W. H. Eisma, "Repeated passive stretching: acute effect on the passive muscle moment and extensibility of short hamstrings," Archives of Physical Medicine and Rehabilitation, vol. 80, no. 4, pp. 407-414, 1999.

[8] N. A. Maffiuletti, "Physiological and methodological considerations for the use of neuromuscular electrical stimulation," European Journal of Applied Physiology, vol. 110, no. 2, pp. 223-234, 2010.

[9] D. Durmuş, G. Alayli, and F. Cantürk, "Effects of quadriceps electrical stimulation program on clinical parameters in the patients with knee osteoarthritis," Clinical Rheumatology, vol. 26, pp. 674-678, 2007.

[10] R. M. Palmieri-Smith, A. C. Thomas, C. Karvonen-Gutierrez, and M. Sowers, "A clinical trial of neuromuscular electrical stimulation in improving quadriceps muscle strength and activation among women with mild and moderate osteoarthritis," Physical Therapy, vol. 90, no. 10, pp. 1441-1452, 2010.

[11] M. R. Maly, P. A. Costigan, and S. J. Olney, "Contribution of psychosocial and mechanical variables to physical performance measures in knee osteoarthritis," Physical Therapy, vol. 85, no. 12, pp. 1318-1328, 2005.

[12] D. Podsiadlo and S. Richardson, "The timed "up and go": a test of basic functional mobility for frail elderly persons," Journal of the American Geriatrics Society, vol. 39, no. 2, pp. 142-148, 1991.

[13] A. A. Wright, C. E. Cook, G. D. Baxter, J. D. Dockerty, and J. H. Abbott, "A comparison of 3 methodological approaches to defining major clinically important improvement of 4 performance measures in patients with hip osteoarthritis," Journal of Orthopaedic and Sports Physical Therapy, vol. 41, no. 5, pp. 319327, 2011.

[14] J. H. Kellgren and J. S. Lawrence, "Radiological assessment of osteo-arthrosis," Annals of the Rheumatic Diseases, vol. 16, no. 4, pp. 494-502, 1957.
[15] S. Shapiro and M. Cameron, "Electrical currents," in Physical Agents in Rehabilitation: From Research to Practice, M. Cameron, Ed., pp. 249-254, Saunders Elsevier Science, St. Louis, Mo, USA, 2003.

[16] J. Felício, Efeito do treinamento aeróbio associado ao treinamento resistido nas variáveis hemodinâmicas, flexibilidade e força em indivíduos hipertensos [Dissertation], Universidade Estadual Paulista, 2008.

[17] J. T. Farrar, J. P. Young Jr., L. LaMoreaux, J. L. Werth, and R. M. Poole, "Clinical importance of changes in chronic pain intensity measured on an 11-point numerical pain rating scale," Pain, vol. 94, no. 2, pp. 149-158, 2001.

[18] N. Bellamy, W. W. Buchanan, C. H. Goldsmith, J. Campbell, and L. W. Stitt, "Validation study of WOMAC: a health status instrument for measuring clinically important patient relevant outcomes to antirheumatic drug therapy in patients with osteoarthritis of the hip or knee," Journal of Rheumatology, vol. 15, no. 12, pp. 1833-1840, 1988.

[19] M. I. Fernandes, Tradução e validação do questionário de qualidade de vida específico para osteoartrose WOMAC (Western Ontario McMaster Universities Osteoarthritis Index) para a língua portuguesa [Dissertation], Universidade Federal de São Paulo, 2003.

[20] H. Chakraborty and H. Gu, A Mixed Model Approach for Intentto-Treat Analysis in Longitudinal Clinical Trials with Missing Values, RTI International, Research Triangle Park, NC, USA, 2009.

[21] J. Cohen, Statistical Power Analysis for the Behavioral Sciences, Erlbaum Associates, Hillsdale, NJ, USA, 1988.

[22] Copyright (c), SAS Institute Inc., Cary, NC, USA, 2002-2008.

[23] M. G. Rosemffet, E. E. Schneeberger, G. Citera et al., "Effects of functional electrostimulation on pain, muscular strength, and functional capacity in patients with osteoarthritis of the knee," Journal of Clinical Rheumatology, vol. 10, no. 5, pp. 246-249, 2004.

[24] T. M. Steffen, T. A. Hacker, and L. Mollinger, "Age- and genderrelated test performance in community-dwelling elderly people: Six-Minute Walk Test, Berg Balance Scale, Timed Up \& Go Test, and gait speeds," Physical Therapy, vol. 82, no. 2, pp. 128-137, 2002.

[25] M. C. Morrissey, "Electromyostimulation from a clinical perspective. A review," Sports Medicine, vol. 6, no. 1, pp. 29-41, 1988.

[26] M. Roig and W. D. Reid, "Electrical stimulation and peripheral muscle function in COPD: a systematic review," Respiratory Medicine, vol. 103, no. 4, pp. 485-495, 2009.

[27] A. M. Imoto, A efetividade da eletroestimulação muscular na reabilitação de tecidos moles do joelho: revisão sistemática $e$ metanálise [Dissertation], Escola Paulista de Medicina, Universidade Federal de São Paulo, 2006.

[28] B. M. Monaghan, B. Caulfield, and D. P. O’Mathúna, “Surface neuromuscular electrical stimulation for quadriceps strengthening pre and post total knee replacement," Cochrane Database of Systematic Reviews, no. 2, Article ID CD007177, 2008.

[29] K.-M. Kim, T. Croy, J. Hertel, and S. Saliba, "Effects of neuromuscular electrical stimulation after anterior cruciate ligament reconstruction on quadriceps strength, function, and patientoriented outcomes: a systematic review," Journal of Orthopaedic and Sports Physical Therapy, vol. 40, no. 7, pp. 383-391, 2010.

[30] L. A. Talbot, J. M. Gaines, S. M. Ling, and E. J. Metter, "A homebased protocol of electrical muscle stimulation for quadriceps muscle strength in older adults with osteoarthritis of the knee," Journal of Rheumatology, vol. 30, no. 7, pp. 1571-1578, 2003. 
[31] P. Z. Nigri, M. S. Peccin, G. J. D. M. Almeida, and M. Cohen, "Translation, validation and cultural adaptation of the "activities of daily living" scale (ADLS)," Acta Ortopedica Brasileira, vol. 15, no. 2, pp. 101-104, 2007.

[32] F. C. Marx, L. M. de Oliveira, and C. G. Bellini, "Tradução e Validação Cultural do Questionário Algofuncional de Lequesne para Osteoartrite de Joelhos e Quadris para a Língua Portuguesa," Revista Brasileira de Reumatologia, vol. 46, no. 2, pp. 253-260, 2006.

[33] D. Moher, S. Hopewell, K. F. Schulz et al., "CONSORT 2010 explanation and elaboration: updated guidelines for reporting parallel group randomised trials," Journal of Clinical Epidemiology, vol. 63, pp. 1-37, 2010. 


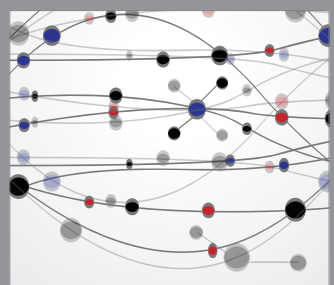

The Scientific World Journal
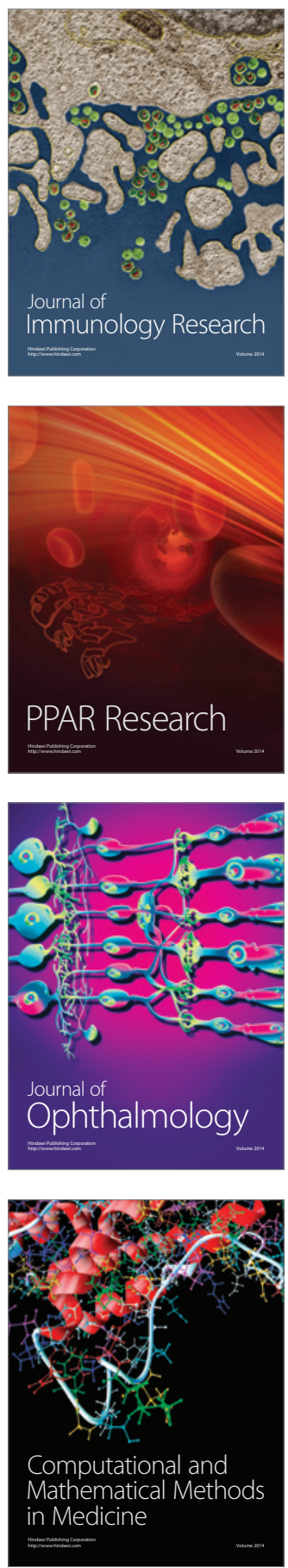

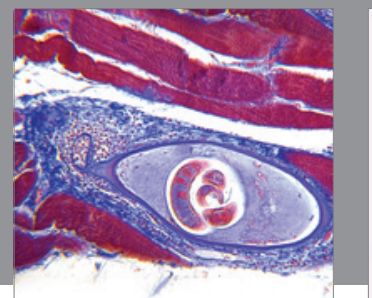

Gastroenterology

Research and Practice
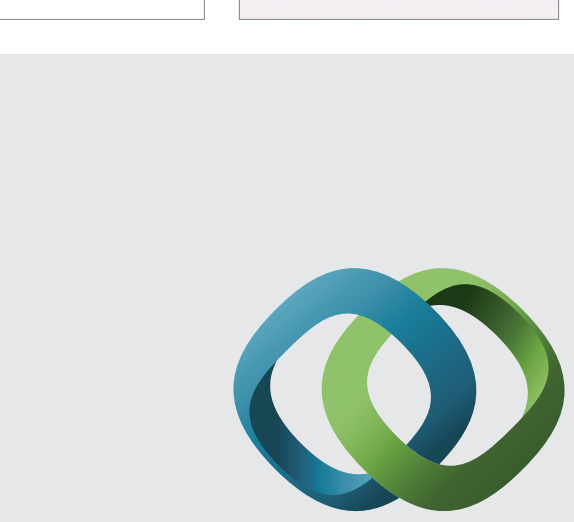

\section{Hindawi}

Submit your manuscripts at

http://www.hindawi.com
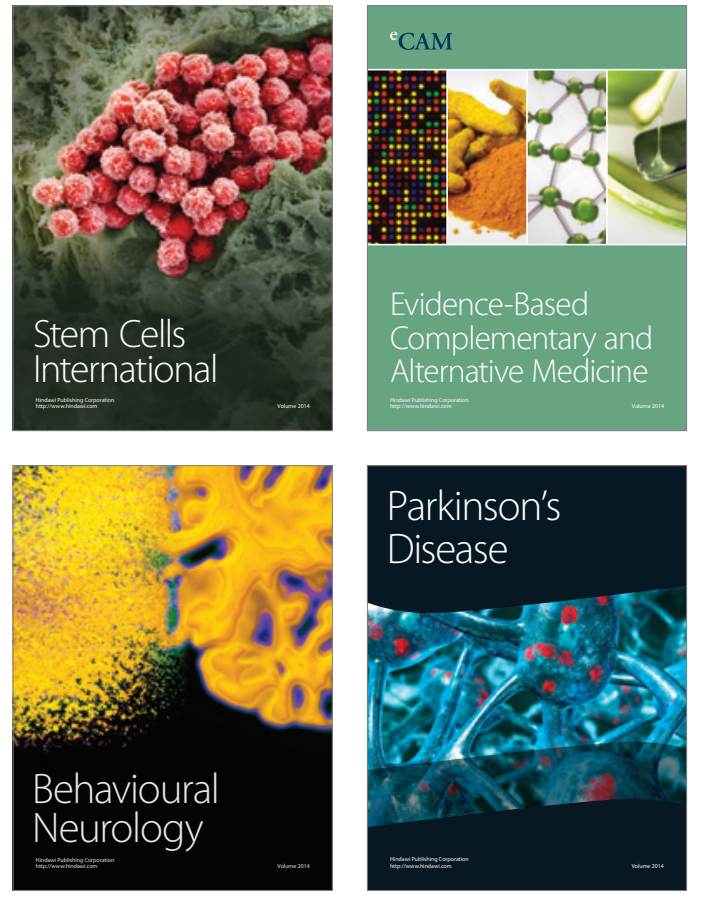
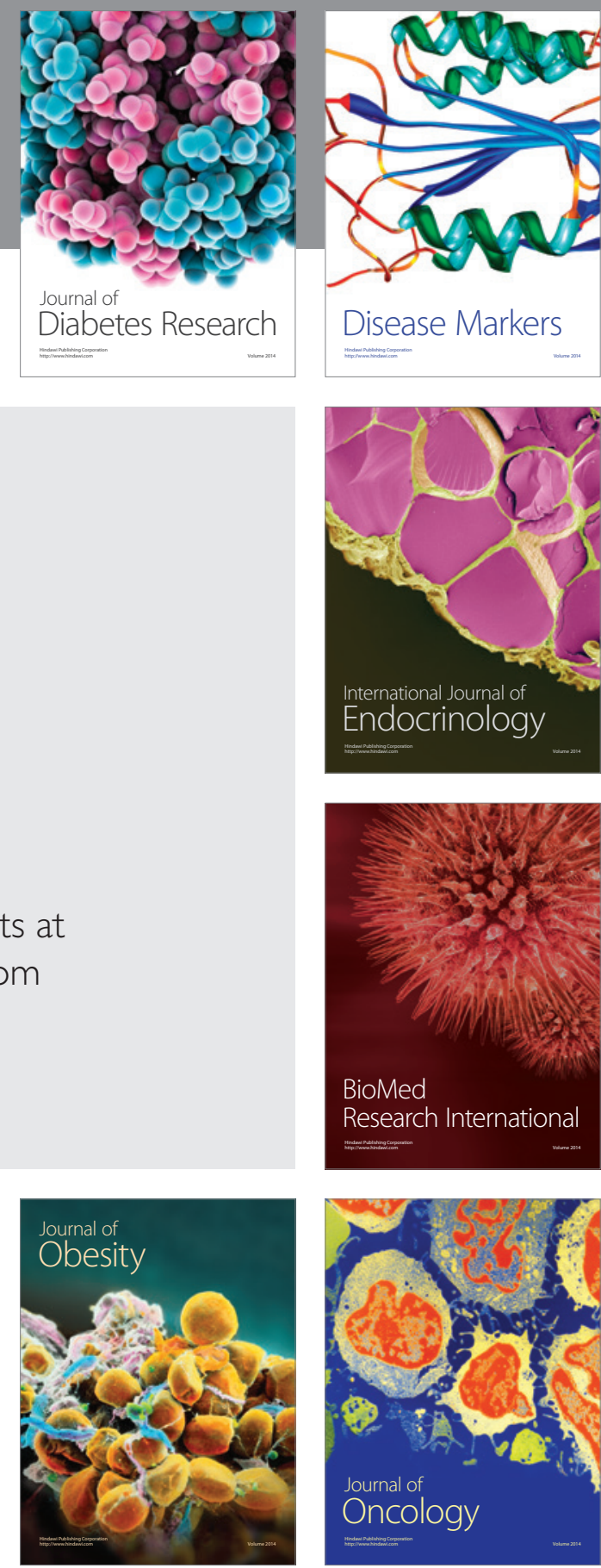

Disease Markers
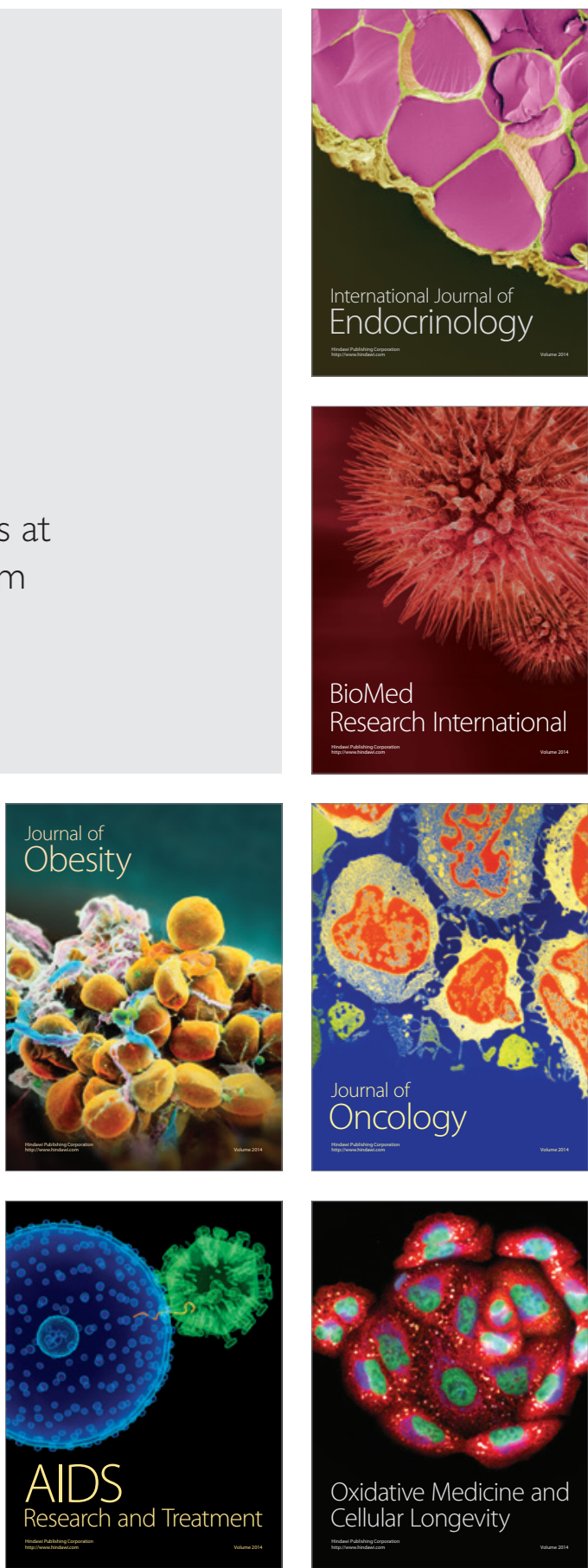